\title{
Variations in the magnitude of attentional capture: Testing a two-process model
}

\author{
Brian A. Anderson ANd Charles L. Folk \\ Villanova University, Villanova, Pennsylvania
}

\begin{abstract}
Although large variations in the magnitude of attentional capture have been evidenced across a wide range of studies and paradigms (see Burnham, 2007, for a review), the nature of these variations is unclear. In the present study, we used a modified spatial cuing task to address two related issues. In the first experiment, we explored the hypothesis that the magnitude of attentional capture varies systematically as a function of cuetarget similarity. Targets of a particular color were preceded by uninformative peripheral cues carrying varying percentages of the target color. As was predicted, the magnitude of attentional capture varied directly with the similarity between cue and target. In the second experiment, we explored whether these similarity effects reflect a mixture of trials on which attention is fully captured and trials on which attention is not captured at all (i.e., a two-process model). A mixture analysis conducted on obtained reaction time distributions proved inconsistent with a two-process model.
\end{abstract}

Given the informational demands of the visual environment, combined with limitations on attentional resources, a crucial task of the visual perceptual system is to selectively allocate limited attentional resources to elements of the visual field that are most critical to current behavioral goals. The hypothetical mechanism that accomplishes this task is referred to as selective attention. One goal of research on selective attention has been to characterize the processes by which attentional resources are allocated. This research has firmly established that attention allocation can result either from the voluntary push of attention to relevant locations or objects (e.g., Posner, 1980) or from the involuntary pull of attentional resources by salient stimuli, a phenomenon known as attentional capture (e.g., Jonides, 1981; Yantis \& Jonides, 1984).

A number of paradigms have been developed to study attentional capture. In the irrelevant singleton paradigm, participants search for a target in a display that contains a singleton in some feature property, such as abrupt onset or color. Attentional capture is reflected in flat search slopes for targets that happen to appear at the singleton location (Yantis \& Jonides, 1984). In the additional singleton paradigm, participants search for a singleton target in displays in which one of the nontargets is also a singleton in a different feature dimension. Capture is assumed if response times (RTs) are longer in the presence of this distractor than when no singleton distractor appears in the display (e.g., Theeuwes, 1992). In the modified spatial cuing paradigm, target search displays are preceded by a cue display containing a singleton whose location is uncorrelated with the location of the subsequent target. Capture is present if there are shorter RTs when the target appears at the cued location than when it appears at an uncued location (valid and invalid cue trials, respectively; e.g., Folk, Remington, \& Johnston, 1992). Finally, capture has also been studied using a rapid serial visual presentation (RSVP) paradigm, in which participants search for a target in a stream of characters appearing at fixation. Capture of spatial attention is reflected in a reduction in the accuracy of target detection associated with the presentation of irrelevant singletons in the periphery (e.g., Folk, Leber, \& Egeth, 2002).

The results of these different paradigms have not always converged, which has led to fundamentally different theoretical perspectives with regard to issues such as the degree to which attentional capture is cognitively penetrable. Primarily on the basis of the results of the additional singleton paradigm, some have argued that attention allocation is prioritized in terms of a strict bottom-up salience ranking (Theeuwes, 1992, 1994). For example, Theeuwes (1992) found that irrelevant additional singletons slowed search down only if they were more salient than the target singleton. However, primarily on the basis of the results of the modified spatial cuing paradigm, others have argued that attentional capture can be modulated by top-down attentional control settings. For example, Folk and Remington (1998) found that color singleton cues produced evidence of attentional capture (i.e., significant cue validity effects) only when they matched the defining color of the subsequent target. Results such as these support the contingent attentional capture (CAC) hypothesis, according to which the capture of attention by a stimulus is contingent on the match between the top-down set adopted by the observer and the defining features of the eliciting stimulus (e.g., Folk et al., 1992; see Duncan \&

C. L. Folk, charles.folk@villanova.edu 
Humphreys, 1989, for a similar notion in the context of visual search).

Another, relatively neglected way in which the results from attentional capture experiments have tended to diverge is in terms of the magnitude of capture effects. In all of the paradigms just mentioned, the capture of attention is measured in terms of a continuous metric (RTs or accuracy) but is typically defined in terms of a significant difference between discrete conditions. For example, the spatial cuing paradigm defines capture as occurring when nonpredictive cues produce significantly shorter RTs on valid trials than on invalid trials (i.e., a cuing effect), the additional singleton paradigm when distractor-present trials produce significantly longer RTs than do distractor-absent trials, and the RSVP paradigm when trials on which a peripheral distractor is present produce a significant decrement in target report relative to no-distractor trials.

A recent review of the attentional capture literature provided by Burnham (2007) demonstrates that the many documented instances of captured attention vary considerably in terms of the magnitude of measures used, both across and within studies. For example, in the context of the additional singleton paradigm, Theeuwes, De Vries, and Godijn (2003) observed significant distractor effects of 20 and $208 \mathrm{msec}$ in a single study, using distractors defined by either one or two randomly varying color values, respectively. In the context of spatial cuing, Folk, Remington, and Wright (1994, Experiment 3) observed a 43msec cuing effect when the target and cues were defined by apparent motion; however, when the target and cues were defined by specific color values, a cuing effect of more than twice that value was observed (Folk \& Remington, 1998, Experiment 1). Using the RSVP paradigm and arguing for the mediating role of stimulus-driven factors in top-down attentional control, Lamy, Leber, and Egeth (2004) reported two instances of captured attention that, themselves, differed to a statistically significant degree.

What might account for such large variations in the magnitude of capture effects, and to what extent might these variations be theoretically meaningful? With respect to the latter question, we note an analogous situation in the visual search literature. In early work in which the visual search paradigm was used, a significant display-size-RT slope was taken as evidence of serial search and the lack of a statistically significant slope as evidence of parallel search (e.g., Treisman \& Gelade, 1980). However, close analysis of significant variations in slope across conditions and experiments has led to significant theoretical advances, such as the guided search model of Wolfe, Cave, and Franzel (1989). Thus, in the case of attentional capture, understanding variations in the magnitude of capture may also yield important theoretical insights.

With respect to the question of what might account for such variations, there are two related issues to consider. First, there is the issue of what kinds of factors influence the magnitude of attentional capture. For example, according to strictly bottom-up, saliency-based models of capture (Theeuwes, 1992), one might expect the magnitude of capture to vary with the relative salience of the eliciting stimulus. Alternatively, according to contingency-based models, one might expect the magnitude of capture to vary with the similarity between the defining features of the eliciting stimulus and the current top-down set of the observer. Indeed, there is some evidence for such similarity effects in attentional capture. For example, Ansorge and Heumann (2003) found significant cue validity effects with cues that were similar to, but did not exactly match, the target color. In addition, Pratt and McAuliffe (2002) found significant cue validity effects with cues that matched the target on a defining feature (e.g., color) but differed from the target on a nondefining feature (i.e., whether it was an onset singleton). Thus, although the cues did not exactly match the target, they nonetheless produced evidence of capture. These previous researchers, however, did not directly compare the magnitude of capture by similar versus matching cues, nor have they explored the effect of varying the degree of similarity on the magnitude of capture. One purpose of the present study is to provide a systematic exploration of such color similarity effects in attentional capture.

A second issue to consider is the nature of the mechanism by which such factors result in variations in the magnitude of capture. Again, there would appear to be at least two possibilities. The first is that factors such as salience and similarity might produce continuous variations in the amount of resources allocated to the eliciting stimulus, such that more salient or more similar stimuli elicit more attentional resources and thus produce a larger magnitude of capture. In this sense, different degrees of the same process occur consistently over trials. This can be termed a continuous model of attentional capture. The second possibility is that the process by which attention is captured is all or none, in that either all available resources are elicited or none are, but the frequency (i.e., the percentage of trials) with which these two alternatives occur varies with changes in saliency or similarity. That is, variations in the magnitude of attentional capture might reflect various combinations of trials on which attention is captured and trials on which attention is diffuse and not captured, resulting in mixture distributions. When attention is captured relatively less often by a stimulus, the magnitude of attentional capture (as measured by mean differences across trials) will decrease proportionally. This can be termed a two-process model of attention allocation (Jonides, 1983). A second purpose of the present study is to distinguish between continuous and two-process accounts.

The mixture distribution predicted by a two-process model provides a basis by which to differentiate between these continuous and two-process accounts. In order to generate the predicted mixture distribution for each participant, samples of trials on which attention is thought to be maximally captured (i.e., captured on every trial minus random variation) and trials on which attention is thought to be completely diffuse (i.e., uncaptured) must first be obtained. These are termed basis distributions (Yantis, Meyer, \& Smith, 1991). Then, an RT distribution whose mean falls between the means of the basis distributions (i.e., an intermediate distribution) must also be obtained. 
An iterative algorithm is then applied that systematically samples mixture combinations from the basis distributions to find the predicted mixture distribution that most closely resembles the obtained intermediate distribution (i.e., that minimizes $\chi^{2}$ ). Statistical characteristics of this intermediate distribution can then be compared with the characteristics of the best-fitting mixture distribution predicted from the basis distributions. If the two differ significantly, the two-process model is disconfirmed. For example, suppose the mean variance of the obtained intermediate distributions across participants is significantly less than the mean predicted variance. This would suggest that variations in the magnitude of attentional capture across conditions do not reflect different percentages of capture and no-capture trials but, rather, reflect a continuous change in the nature of attentional capture on each trial (e.g., variations in the amount of resources allocated).

Previous researchers have successfully compared a twoprocess model with a continuous model of attention allocation in the context of voluntary shifts of attention. It is well documented that the magnitude of cuing effects with central or voluntary cues varies systematically with the predictive validity of the cue, such that cues with greater validity produce larger cuing effects (e.g., Eriksen \& Yeh, 1985; Jonides, 1980). Maximally captured attention can be defined as occurring in the context of a $100 \%$ valid cue and diffuse attention when all possible target locations are simultaneously cued. Johnson and Yantis (1995) used these two conditions to generate basis distributions of fully focused and fully diffuse attention, respectively. They also ran a condition in which the cue was $50 \%$ valid in order to generate an intermediate distribution. When the best-fitting mixture distribution was compared with the observed intermediate distribution, a significant difference emerged across participants. Thus, the data were inconsistent with a two-process model and the mixture distributions that it predicts (note that Eriksen \& Yeh, 1985, reached a similar conclusion).

In the context of involuntary attention allocation, there has been only one published application of a mixture analysis. Theeuwes (1990, Experiment 3) applied the mixture analysis logic to data from the irrelevant singleton paradigm in which search slopes for targets appearing inside an irrelevant form singleton were reduced relative to control conditions in which no irrelevant singleton appeared. Specifically, search performance in this condition was modeled in terms of a mixture of trials on which the singleton fully captured attention and trials on which it did not capture attention at all. The results provided tentative support for a two-process model, suggesting that salient singletons may indeed result in capture that occurs on a proportion of trials. However, there have been no published studies in which a mixture analysis has been applied to variations in capture magnitude associated with the similarity between cue and target, which are generally assumed to reflect top-down modulation. Thus, characterizing the mechanisms underlying such effects has important implications for modeling the mechanisms by which top-down set modulates the capture of attention.
Applying a mixture analysis to involuntary attention allocation, however, is not as straightforward as in the case of voluntary attention allocation. For example, in the context of the spatial cuing paradigm, basis distributions cannot be obtained by varying cue validity, because in order to attribute cuing effects to involuntary attention allocation, cues must remain completely nonpredictive. Thus, there must be some other means of producing systematic variations in the magnitude of attentional capture that can reasonably be attributed to maximally captured, fully uncaptured, and intermediate states.

Research on CAC suggests a means of obtaining the necessary basis and intermediate distributions needed to perform a mixture analysis. The CAC hypothesis posits that a cue matching the target on a defining property produces the optimal circumstance for attention to be captured. Likewise, according to CAC, cues whose defining feature value is orthogonal to the defining feature of the target should produce no capture at all. Studies such as Folk and Remington (1998) support these assumptions, in that color singleton cues that matched the color of the target (e.g., red cue, red target) produced large $(\sim 100-\mathrm{msec})$ and highly significant cue validity effects, whereas cues that did not match the target (e.g., red cue, green target) produced nonsignificant cue validity effects which hovered around zero. Thus, varying the similarity between the defining feature of the target and cue may provide an appropriate intermediate RT distribution with which to conduct a mixture analysis and thereby to assess whether variations in the magnitude of attentional capture are best accounted for in terms of a continuous model or a twoprocess model.

The purpose of Experiment 1 was to test whether systematic manipulation of the similarity between cue and target in the modified spatial cuing paradigm used by Folk and Remington (1998) would indeed yield systematic variation in the magnitude of attentional capture as measured by the size of cue validity effects. A target defined by one of two possible colors (red or green) was paired with cues that varied in similarity to the target in terms of the percentage of the two colors $(100 \%$ red $/ 0 \%$ green, $66 \%$ $\mathrm{red} / 33 \%$ green, $50 \% \mathrm{red} / 50 \%$ green, $33 \% \mathrm{red} / 66 \%$ green, and $0 \% \mathrm{red} / 100 \%$ green). It was predicted that the magnitude of attentional capture would vary directly with the percentage of target color present in the cue, such that the two extremes could be used as basis distributions for maximally captured and fully uncaptured states and the mixed cues as potential intermediate mixture distributions.

\section{EXPERIMENT 1}

\section{Method}

Participants. Twenty undergraduate students were recruited from the Villanova University human participant pool. All were screened for normal or corrected-to-normal visual acuity and color vision. The participants were compensated for their time with credit toward fulfillment of a class research requirement.

Apparatus. A Dell Workstation PSW370 equipped with E-Prime software was used to present the stimuli on a Dell 2000FP monitor. The participants viewed the monitor from a distance of approximately $50 \mathrm{~cm}$, in a dimly lit room. 


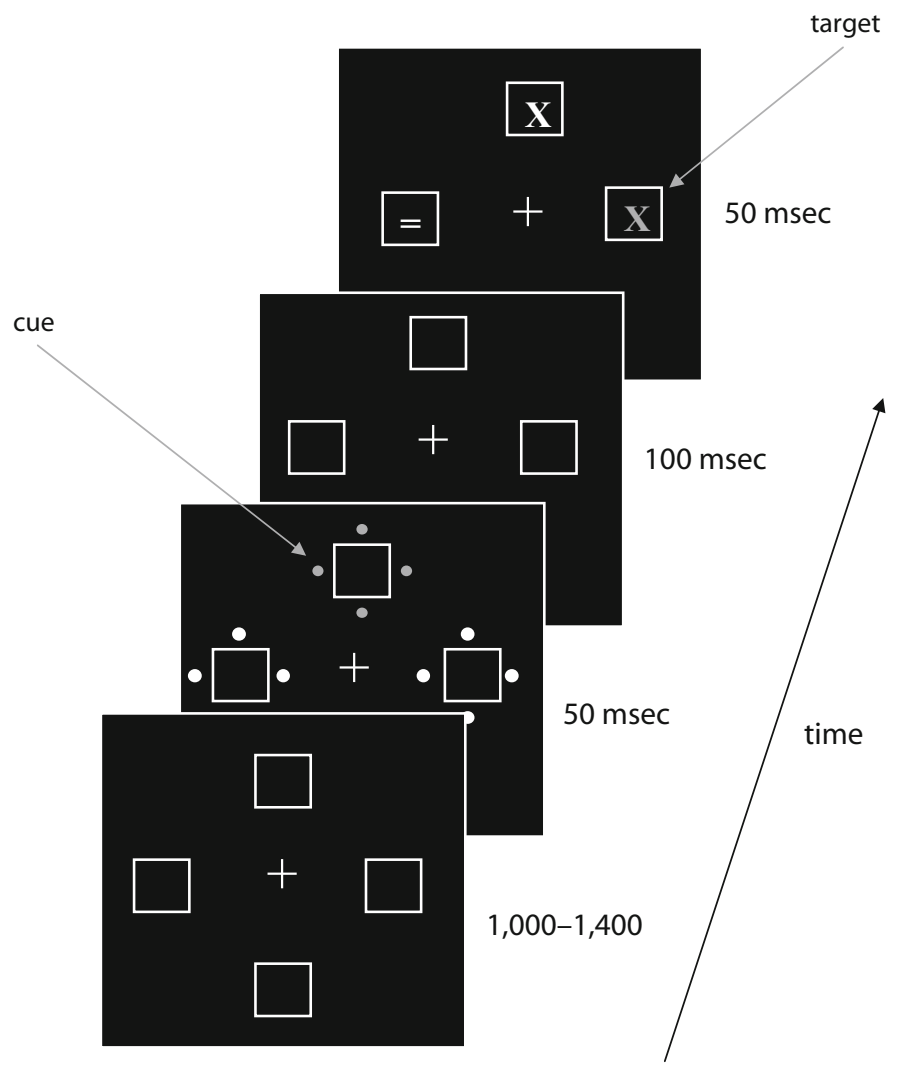

Figure 1. Sequence of events and time course of a trial. Targets were either red or green, manipulated between participants, and cues were $100 \%$ red, $66 \%$ red $/ 33 \%$ green, $50 \%$ red $/ 50 \%$ green, $33 \%$ red $/ 66 \%$ green, or $100 \%$ green, manipulated within participants.

Stimuli. Each trial involved three different displays, which were designed using MS Paint software. An example of each, along with their sequence of presentation and time course, is shown in Figure 1. The first display, the fixation display, consisted of a white fixation square $\left(0.34^{\circ} \times 0.34^{\circ} ;\right.$ RGB: $255,255,255 ;$ CIE [Yxy]: $\left.100, .31, .33\right)$ surrounded by four white peripheral boxes $\left(1.15^{\circ} \times 1.15^{\circ}\right)$ placed $4.1^{\circ}$ above, below, to the left, and to the right of fixation on a black background (RGB $0,0,0)$. The boxes remained on the screen throughout the course of a trial.

The second display, the cue display, consisted of the appearance of four sets of small circles $\left(0.23^{\circ}\right.$ in diameter $)$ surrounding each of the four peripheral boxes in a diamond configuration. Three sets of the circles were white, whereas the other set was one of five possible colors. The colors used were red (RGB: 255, 0, 0; CIE [Yxy]: 21.3, .64, .33); green (RGB: 0, 255, 0; CIE [Yxy]: 71.5, .30, .60); and three blendings of these two colors containing $33 \%$ green and $66 \%$ red (RGB: 170, 85, 0; CIE [Yxy]: 15.0, .54, .41), 50\% green and $50 \%$ red (RGB: 127, 127, 0; CIE [Yxy]: 19.7, .42, .51), and $66 \%$ green and $33 \%$ red (RGB: 85, 170, 0; CIE [Yxy]: 30.7, .34, .57). Note that in defining the various colors used for these stimuli, luminance, as well as hue, varied substantially, with higher luminance for green than for red colors (see the Discussion section below regarding this point).

The final display, the target display, consisted of the appearance of an $\mathrm{X}$ or an $=$ in each of the peripheral boxes. These characters subtended approximately $0.57^{\circ}$ in height and width. Like the circles, three of the characters were white, whereas the other was colored. Half of the participants saw red targets, and the other half saw green targets.
Design. The experiment consisted of three blocks of 160 trials. Within each block, all five cue colors were presented equally often. Each target character (i.e., a colored $\mathrm{X}$ or $=$ ) appeared equally often at each of the four possible locations within each condition, whereas the identity of the nontarget characters were chosen randomly on each trial, with the constraint that two of each character appear in every trial. Like the target characters, the colored cue circles appeared equally often at each of the four possible locations. In addition, the location of the cue was uncorrelated with the target location. For each cue-target color combination, the cue appeared at the same location as the target on $25 \%$ (8) of the trials and at a different location on $75 \%$ (24) of the trials within a block. Finally, for trials on which the cue location and the target location were different, the cue appeared in each of the three nontarget locations equally often for each possible target location.

Procedure. Each participant was tested individually over the course of a single 50-min session. Each session took place inside a dimly lit laboratory room. The experimenter familiarized all of the participants with the task by providing written and oral descriptions of the stimuli and procedures. The participants were instructed to respond as quickly as possible, while minimizing errors. Maintaining fixation on the central square was highly stressed, and the participants were told that failing to do so impairs overall performance. The participants were also fully informed of the relationship between cue location and target location and told to try to ignore the cue.

Each trial began with a 500-msec presentation of the fixation display. After this $500-\mathrm{msec}$ period, the fixation square blinked off for $100 \mathrm{msec}$ and then back on for a randomly varying foreperiod of $1,000,1,100,1,200$, or $1,400 \mathrm{msec}$. The cue display then appeared 


\section{A}

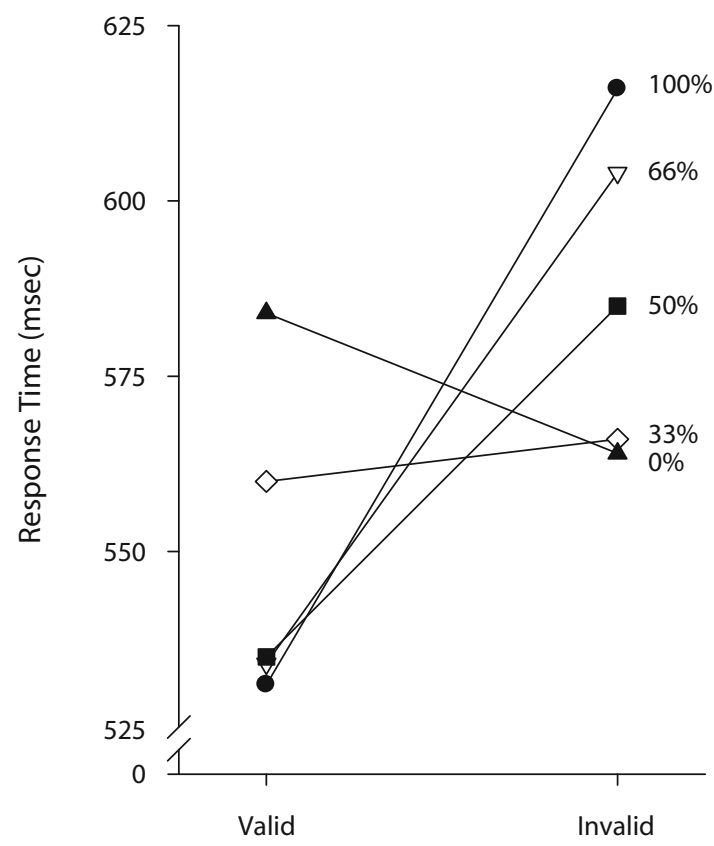

Cue Validity

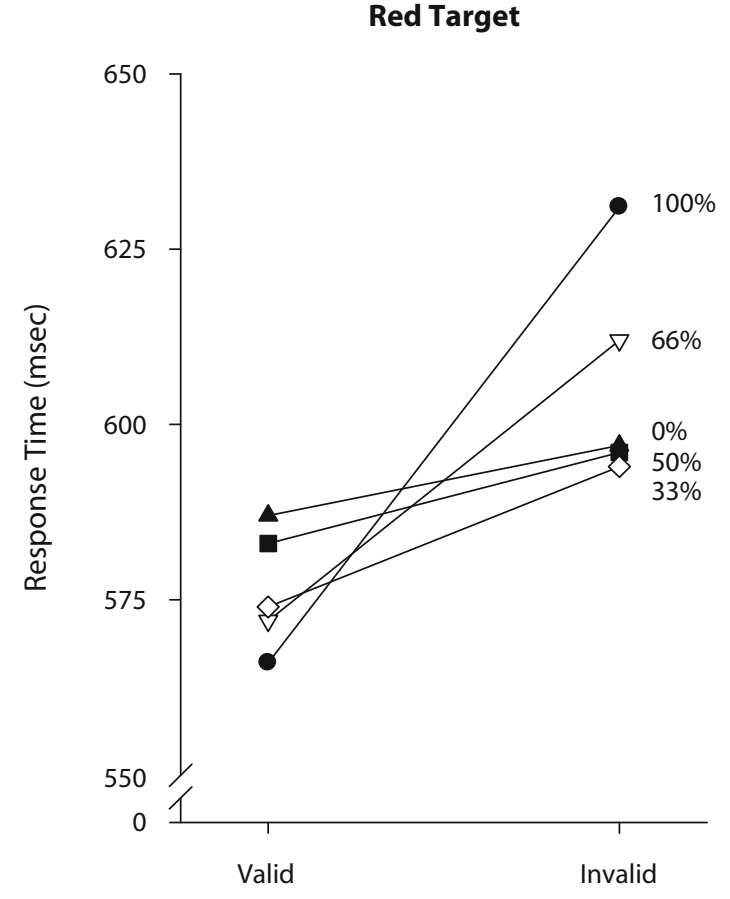

Cue Validity
B

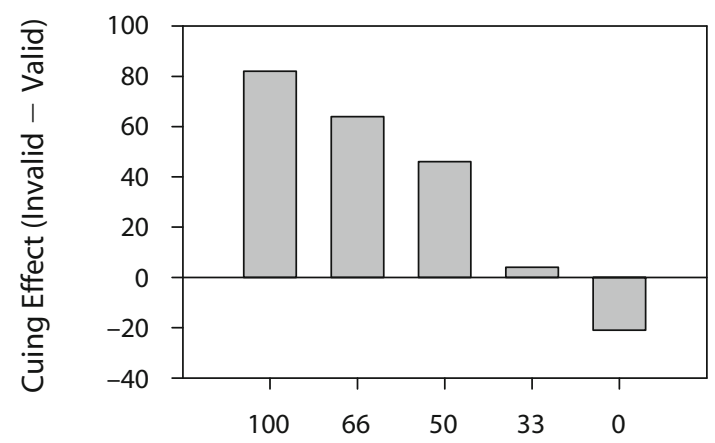

\% Target Color

Green Target

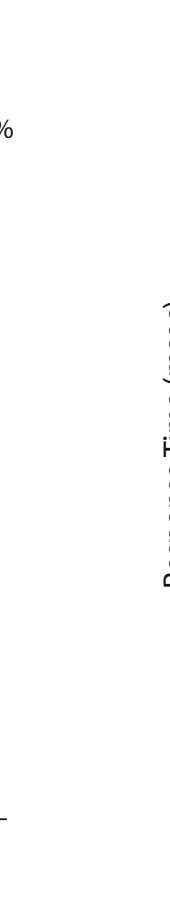


Table 1

Error Rates (Percent) As a Function of Target Color (Green,

Red), Cue Color (100\%, 66\%, 50\%, 33\%, $0 \%$ of Target Color), and Cue Validity (Valid, Invalid) in Experiment 1

\begin{tabular}{cccccc}
\hline & \multicolumn{5}{c}{ Cue Color } \\
\cline { 2 - 6 } Cue Validity & $100 \%$ & $66 \%$ & $50 \%$ & $33 \%$ & $0 \%$ \\
\hline \multirow{5}{*}{ Green Target } \\
Valid & 2.1 & 2.9 & 1.3 & 5.0 & 2.5 \\
Invalid & 3.8 & 3.3 & 3.3 & 3.5 & 2.5 \\
& \multicolumn{5}{c}{ Red Target } \\
Valid & 1.3 & 2.1 & 1.3 & 1.3 & 2.9 \\
Invalid & 2.9 & 3.6 & 3.1 & 3.5 & 2.5 \\
\hline
\end{tabular}

rates in Table 1 . These data were subjected to a $5 \times 2$ ANOVA, with cue color (100\% target color, $66 \%$ target color, 50\% target color, 33\% target color, and $0 \%$ target color) and cue validity (valid and invalid) as withinsubjects variables. Although the general pattern of results was consistent across target colors, the three-way interaction was, in fact, significant $[F(4,72)=6.274, p<.001]$. Therefore, the results of both target colors are reported. The main effect of cue validity was significant for both green and red targets $[F(1,9)=23.239, p=.001$, and $F(1,9)=12.047, p=.007$, respectively $]$, as was the interaction between cue validity and cue color for both target colors $[F(4,36)=20.862, p<.001$, and $F(4,36)=6.695$, $p<.001$, respectively]. The significant interactions confirm what is evident in the figure: The magnitude of cue validity effects varied as a function of the percentage of the target color contained in the cue.

To explore this effect more fully, simple effects analyses of cue validity were conducted on each cue color condition for each target color. For green targets, significant validity effects were found for the $100 \%, 66 \%$, and $50 \%$ conditions $[t(9)=-5.136, p=.001 ; t(9)=-6.082, p<.001$; and $t(9)=-3.542, p=.006$, respectively]. In addition, a significant negative cue validity effect was found for the $0 \%$ target-color cue condition $[t(9)=3.984, p=.003]$. For red targets, significant cue validity effects were found only in the $100 \%$ and $66 \%$ conditions $[t(9)=-7.277$, $p<.001$, and $t(9)=-4.114, p=.003$, respectively].

The differences in RTs between valid and invalid trials for each cue color were also subjected to a linear trend analysis, using proportion of the target color as a weight. This analysis revealed a significant linear trend for both the green and red target color conditions $[F(1,45)=58.160, p<.001$, and $F(1,45)=38.766, p<.001$, respectively], meaning that the participants showed increasingly smaller cuing effects as the cue color contained less and less of the target color.

The same ANOVA was conducted on error rates to ensure that the results of the aforementioned analyses were not contaminated by a speed-accuracy trade-off. This analysis revealed no significant main effects or interactions for either target color condition. Thus, there was no evidence of a speed-accuracy trade-off.

\section{Discussion}

There are several important findings in Experiment 1. First, consistent with previous findings concerning CAC, a cue-target match (i.e., the $100 \%$ cue condition) produced a strong cuing effect, whereas a discrete cue-target mismatch (i.e., the $0 \%$ cue condition) produced no evidence of capture. Also, consistent with the findings of Ansorge and Heumann (2003), blended cue colors were found to produce attentional capture as well. The present results go further, however, to show that the magnitude of cue validity effects vary quite systematically with the similarity between the cue and target, operationalized in terms of the percentage of target color present in the cue. Although this effect was strongest in the green target condition, the same general pattern was observed for both target colors, as was evidenced by a significant linear trend in both cases.

One might argue that, given that the colors used in this experiment varied not only in terms of hue, but also in terms of luminance, the variations in the magnitude of capture observed in the present experiment may be related to bottom-up stimulus salience (driven by luminance) rather than color similarity. The full pattern of data, however, provides strong evidence against this possibility. Specifically, if capture were driven by the relative luminance of the cues, cues with more green in them (i.e., of higher luminance) should have always produced larger cuing effects. Instead, the order of the magnitude of cuing effects associated with specific cue colors when paired with one target color was generally reversed when the exact same cues were paired with targets of the other color (note that this reversal is not apparent in the figures, because cues were coded in terms of the percentage of the target color for that target condition). Thus, the obtained cuing effects must reflect variations associated with the similarity between a given cue and the top-down set associated with the target color, rather than the relative bottom-up salience (i.e., luminance) of a given cue. This is not to say that relative salience never influences the magnitude of attentional capture, but simply that bottom-up salience cannot account for the variations observed in the present experiment.

Finally, note that for green targets, the $0 \%$ cue condition actually produced a significant negative cuing effect. Although the precise nature of this effect and why it only occurred in the green target condition is unclear, there is some precedent in the literature for reverse cuing effects in the modified spatial cuing paradigm (e.g., Folk \& Remington, 2008; Lamy et al., 2004), and some have suggested that it reflects a form of location-specific inhibition similar to inhibition of return.

In summary, the results of Experiment 1 establish that the magnitude of attentional capture as measured by cue validity effects in the modified spatial cuing paradigm vary systematically as a function of the similarity between the target color and cue color. In Experiment 2, we addressed the issue of whether these similarity effects can best be accounted for in terms of a continuous or twoprocess model, by applying a mixture analysis.

\section{EXPERIMENT 2}

As was stated previously, a mixture analysis assesses the probability that a distribution falling intermediate be- 
tween two basis distributions represents a sampling of trials from the basis distributions or a mixture of these distributions. When the probability falls below some criterion, such as that for statistical significance, the null hypothesis that the intermediate distribution and the predicted mixture distribution do not differ can be rejected. Such a result would disconfirm a two-process model. One way to perform a mixture analysis is to compare the variability predicted by a mixture distribution with the observed variability of an intermediate distribution using a paired-samples $t$ test. The variability predicted by a mixture distribution follows logically from the means and variances of the basis distributions and the maximum likelihood mixing probabilities, which are determined by an iterative algorithm (Yantis et al., 1991). The maximum likelihood mixing probabilities reflect the relative contribution of each basis distribution that produces a hypothetical mixture distribution most closely resembling the observed intermediate distribution. In short, the algorithm categorizes the observed intermediate RT distribution into bins and then iteratively generates hypothetical distributions using adaptively varying mixing probabilities until the $\chi^{2}$ value between the observed and predicted distribution is minimized. Evidence for the effectiveness of this algorithm in hypothesis testing has been obtained through computer simulation (Yantis et al., 1991). The mixing probabilities obtained from the iterative algorithm provide the weights for the basis distributions, and a weighted average of their variances is obtained. Then, an inflation factor that takes into account the distance between the two basis distributions as a function of the mixing probabilities is added to this weighted average. Given two basis distributions, the variance predicted by a mixture distribution is determined using the following equation, in which $\alpha$ equals the relative contribution of basis distribution $[\mathrm{x}]$ as represented by its mixing probability:

$$
\sigma_{\text {mixture }}^{2}=\alpha_{1} \sigma_{1}^{2}+\alpha_{2} \sigma_{2}^{2}+\alpha_{1} \alpha_{2}\left(\mu_{1}-\mu_{2}\right)^{2} \text {. }
$$

For example, if the intermediate distribution of interest was best represented by an equal contribution of the two basis distributions, the variances of which were $10,000 \mathrm{msec}^{2}$ each and the means of which were 400 and $500 \mathrm{msec}$, the variance of the intermediate distribution predicted by a mixture of the two basis distributions would be as follows:

$$
\begin{aligned}
(.5)(10,000) & +(.5)(10,000) \\
& +(.5)(.5)(500-400)^{2}=12,500 \mathrm{msec}^{2} .
\end{aligned}
$$

As would be expected, the variance predicted by a mixture distribution is typically greater than the variance of the two contributing basis distributions. Once determined, the variance predicted by a mixture distribution can be compared with the actual variance of the intermediate distribution for each participant using a paired-samples $t$ test. In the event that the actual variance is significantly lower than the variance predicted by a mixture distribution, the two-process model and the mixture distributions that it predicts can be confidently rejected.

In a spatial cuing paradigm, the magnitude of attentional capture is defined as the difference between the mean
RTs on valid and invalid trials. The distributions specific to either the valid or the invalid trials that comprise this mean difference can be used for a mixture analysis. This is because both types of trials represent the influence of attentional capture, just in different directions. That is, as attentional capture increases as a function of cue-target similarity, RTs on valid trials should decrease and RTs on invalid trials should increase. Note that in Experiment 1, the means of both of these types of trials produced systematic variation relative to cue-target similarity.

Several factors affect the statistical power of a mixture analysis or its ability to discriminate between the predicted mixture distribution and the observed intermediate distribution (Yantis et al., 1991). The statistical power of a mixture analysis is greatest when the intermediate distribution is distinct or sufficiently separated from the basis distributions. In this sense, the intermediate distribution should ideally fall equidistant between the two basis distributions. Also, the statistical power of a mixture analysis is greatest when the variability of the basis distributions is minimized. Finally, as with all statistical tests, more observations produce greater statistical power. The maximum likelihood approach to mixture analyses is important, because it uses empirical, rather than theoretical, means to determine the relative contribution of the basis distributions and accounts for participant-by-participant variation. Given this maximum likelihood approach, however, it is especially important to give attention to the circumstances optimal to maximizing statistical power.

Experiment 2 is a replication of Experiment 1 that was designed to maximize the statistical power of the mixture analysis. This was accomplished in several ways. First, given that green targets produced more systematic variation in capture in Experiment 1, all of the participants in Experiment 2 searched for green targets. In addition, given that the $50 \%$ target-color cue condition produced mean RTs that fell approximately halfway between the $100 \%$ and $0 \%$ target-color cue conditions, only this condition was included as an intermediate target-similarity condition. Finally, invalid trials were selected to be used for the mixture analysis over valid trials for three reasons: (1) Mean RTs on invalid trials were farther apart than the means on valid trials, (2) invalid trials provide three times as many observations, and (3) invalid trials are immune to potential position-specific inhibitory effects that could influence RTs on valid trials (e.g., the reverse cuing effects observed for the $0 \%$ cue condition in Experiment 1).

\section{Method}

Participants. Thirty-two undergraduate students were recruited from the Villanova University human participant pool. All were screened for normal or corrected-to-normal visual acuity and color vision. The participants were compensated for their time with credit toward fulfillment of a class research requirement. None of the participants had participated in Experiment 1.

Apparatus and Stimuli. The apparatus and stimuli were identical to those used in Experiment 1, with two exceptions. Two cue colors were removed, leaving only the $100 \%, 50 \%$, and $0 \%$ target-color cue conditions. In addition, only green targets were used.

Design and Procedure. The design and procedure were identical to those in Experiment 1, with two exceptions. Only three cue colors 
A

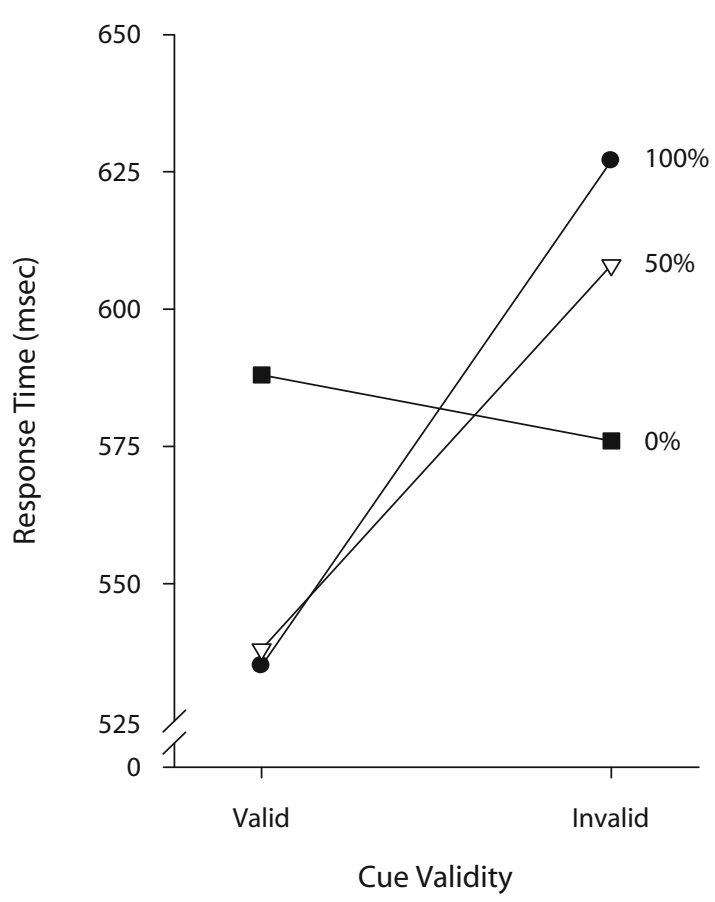

B

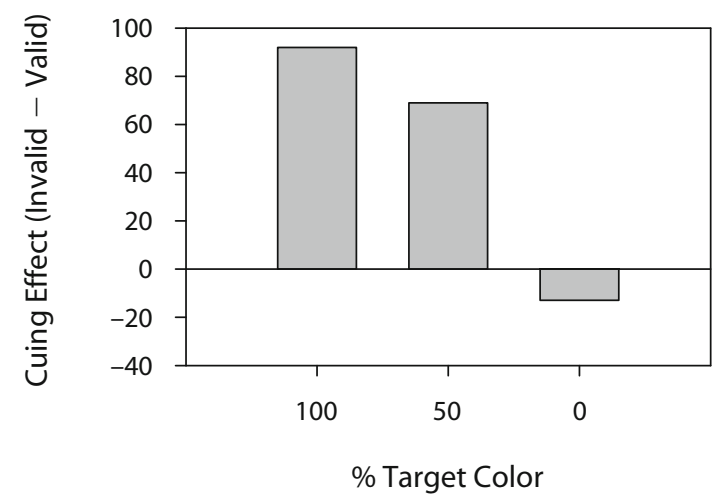

Figure 3. (A) Mean response time as a function of cue validity and percentage of target color contained in the cue in Experiment 2. (B) Mean cuing effects as a function of percentage of target color contained in the cue for Experiment 2.

were used in each block, creating three different conditions. In addition, the experiment consisted of five blocks of 96 trials.

\section{Results}

Mean RTs for valid and invalid trials as a function of cue color condition are shown in Figure 3 and the error rates in Table 2 . The data were subjected to a $3 \times 2$ ANOVA, with cue color $(100 \%, 50 \%$, and $0 \%$ target color) and cue validity (valid and invalid) as within-subjects variables. Main effects of cue validity and cue color $[F(1,31)=177.398$, $p<.001$, and $F(2,30)=7.489, p=.002$, respectively], as well as an interaction between validity and cue color $[F(2,30)=77.93, p<.001]$, were observed.
Simple effects analyses of cue validity were conducted for each cue color. These comparisons revealed that the $100 \%$ target-color and $50 \%$ target-color cues both captured attention $[t(31)=-16.607, p<.001$, and $t(31)=$ $-12.311, p<.001$, respectively]. The difference between valid and invalid trials for the $0 \%$ target-color cue was also significant, but as in Experiment 1, it was in the opposite direction $[t(31)=2.370, p=.024]$. The differences in RTs between valid and invalid trials by cue color were also subjected to a linear trend analysis, using proportion of the target color as a weight. This analysis revealed a significant trend $[F(1,93)=179.932, p<.001]$, replicating the trend in Experiment 1.

The same ANOVA was conducted on error rates to ensure that the results of the aforementioned analyses were not contaminated by a speed-accuracy trade-off. This analysis revealed only a main effect of cue validity $[F(1,31)=16.506, p<.001]$.

Finally, the data were subjected to a mixture analysis, which compared the statistical properties of an intermediate distribution with those predicted by a best-fitting mixture of the basis distributions representing the discrete capture and no-capture states. Consistent with contingent involuntary orienting accounts, the $100 \%$ and $0 \%$ targetcolor cue conditions were chosen to represent the two basis distributions of maximally captured and diffuse attention, respectively. The mean variance was $10,124 \mathrm{msec}^{2}$ for the former and $9,577 \mathrm{msec}^{2}$ for the latter. The maximum likelihood mixing probabilities were then determined for the $50 \%$ target-color cue condition for each participant using a computer program provided by S. Yantis (personal communication, July 25,2008 ) that executes the iterative algorithm previously discussed. These mixing probabilities were then used to generate the predicted variance for the $50 \%$ target-color condition for each participant according to the formula described above. The predicted and observed variance was then compared across participants using a paired-samples $t$ test. This analysis revealed that the mean observed variance was significantly less than the mean variance predicted by a mixture distribution $(11,073 \mathrm{vs}$. $12,374 \mathrm{msec}^{2}$, respectively) $[t(31)=-2.422, p=.021]$.

As was noted by Yantis et al. (1991), an important assumption for conducting a mixture analysis is that the observations that comprise the basis and intermediate distributions are independent of one another. In the context of the present experiment, this means that in order to avoid systematic bias, the RTs for a trial with a given cue color should be unaffected by the color of the cue on the previous trial. To confirm that the observations in the invalid cue conditions used in the reported mixture analysis were indeed independent, separate one-way ANOVAs were

Table 2

Error Rates (Percent) As a Function of Cue Color (100\%, 50\%, $0 \%$ of Target Color) and Cue Validity (Valid, Invalid) in Experiment 2

\begin{tabular}{cccc}
\hline & \multicolumn{3}{c}{ Cue Color } \\
\cline { 2 - 4 } Cue Validity & $100 \%$ & $50 \%$ & $0 \%$ \\
\hline Valid & 2.0 & 2.7 & 3.8 \\
Invalid & 4.8 & 4.2 & 4.1 \\
\hline
\end{tabular}


conducted for each of the three cue colors (i.e., 100\%, $50 \%$, and $0 \%$ of target color) with previous cue color (i.e., cue color on trial $n-1)$ as the single within-subjects variable. In all three cases, there was no effect of previous cue color (all $\left.F_{\mathrm{S}}<1\right)$.

\section{Discussion}

The results of Experiment 2 replicate those of Experiment 1 very closely with regard to the capture of attention. The $100 \%$ cue produced a large cuing effect, the $0 \%$ cue a small reverse cuing effect, and the $50 \%$ cue a cuing effect that fell between these two extremes. In other words, there was once again systematic variation in the magnitude of the cuing effects associated with the similarity between cue and target color. Most important, when the data were subjected to a mixture analysis, the observed variance for the intermediate cue condition was significantly less than that predicted by a mixture model. Thus, the data from Experiment 2 are inconsistent with a mixture distribution and suggest that the two-process model is not an appropriate characterization of involuntary attention allocation, at least in the context of capture by color singleton cues.

\section{GENERAL DISCUSSION}

The purpose of the present experiments was twofold. First, in these experiments, we sought to explore the effects of cue-target similarity on the magnitude of attentional capture as measured by cue validity effects in the modified spatial cuing paradigm. The second purpose was to test a two-process model of involuntary attention allocation, whereby variations in the magnitude of attentional capture are assumed to reflect differences in the proportion of trials on which attention is fully captured.

With respect to the influence of cue-target similarity, the present results provide strong evidence that the magnitude of attentional capture varies systematically as a function of the percentage of the target color present in the cue. Although previous studies have shown that cues similar to a target can produce attentional capture (e.g., Ansorge \& Heumann, 2003), the present study is the first to demonstrate that the magnitude of attentional capture, as measured by cue validity effects, varies systematically as a function of parametric variations in similarity.

This type of systematic variation also provides the necessary conditions for assessing the presence of a mixture distribution, allowing the opportunity to test whether the two-process model of attention allocation applies to instances of attentional capture. Specifically, RTs for trials on which the cue color exactly matches the target color (and which show the largest cue validity effect) provide a basis distribution reflecting maximal attentional capture, whereas RTs for trials on which the cues carry none of the target color (and which show no, or even a reverse, cue validity effect) provide a basis distribution reflecting no attentional capture. Finally, RTs for trials on which the cue carries a percentage of the target color (and which show an intermediate cue validity effect) provide a distribution with which to test for the presence of mixture of capture and no-capture trials. A mixture analysis applied to the results of Experiment 2 showed that the obtained variance for the intermediate distribution was significantly less than that predicted by a mixture of capture and no-capture trials, disconfirming a two-process model. Thus, the present results suggest that the variations in the magnitude of attentional capture as a function of cue-target similarity found in the existing literature are not accounted for by various combinations of fully captured and not-captured trials across conditions, but, rather, reflect variations in the strength of attentional capture that are consistent across trials.

If variations in the magnitude of attentional capture reflect continuous variation that is relatively consistent across trials, what is the nature of this continuous process? One possibility, as was suggested above, is that cuetarget similarity affects the amount of spatial attentional resources that are drawn to the eliciting stimulus. This is similar to the findings of the literature on voluntary attention allocation, in which variations in the validity of an informative cue yielded evidence of continuous variation in the amount of resources allocated to the cued location (Eriksen \& Yeh, 1985; Johnson \& Yantis, 1995). There is, however, at least one other possibility. Instead of reflecting variations in the amount of allocated resources, cuetarget similarity might produce continuous variation in the speed with which attention is disengaged from the cue (e.g., Theeuwes, Atchley, \& Kramer, 2000). On this account, attention is captured by the cue on every trial (i.e., there are no contingencies in the actual shift of attention), but the higher the percentage of target color contained in the cue (i.e., the greater the similarity to the target), the longer it takes to disengage from the uninformative cue. Thus, variations in the magnitude of attentional capture (i.e., cuing effects) would reflect the degree to which observers have recovered from capture by the time the target display arrives. In terms of the mixture analysis, the basis distributions would reflect fully engaged and fully disengaged trials, and the intermediate distributions would reflect continuous variations in the state of disengagement.

Although continuous variations in the speed of disengagement represent a logically plausible account of the present results, there is mounting behavioral and electrophysiological evidence against the underlying assumption of this account - that attention is captured even by cues that do not share the target property (Eimer \& Kiss, 2008; Folk \& Remington, 2006; Lien, Ruthruff, Goodin, $\&$ Remington, 2008). For example, using behavioral measures, Folk and Remington (2006) found that prime characters presented simultaneously and at the same location as an uninformative cue produced significant cuing effects when the cue color matched the target color but not when the cue color did not match the target color. Thus, unless one assumes that attention is immediately disengaged from the cue without any processing of the simultaneously presented prime, the lack of priming effects for nonmatching cues suggests that spatial attention was never allocated to such cues. Eimer and Kiss and Lien et al. provided electrophysiological evidence consistent with this conclusion by showing that a significant $\mathrm{N} 2 \mathrm{pc}$, which is an ERP component associated with shifts of spatial attention (Kiss, Van Velzen, \& Eimer, 2008), was obtained 
for cues that matched the target, but not for cues that did not match the target. Several other studies have shown the same pattern (i.e., the presence of an N2pc for distractors that match the target and the absence of the N2pc for distractors that did not match the target) in the context of an RSVP paradigm (Kiss, Jolicœur, Dell'Acqua, \& Eimer, 2008; Leblanc, Prime, \& Jolicœur, 2008). Acknowledging that the absence of an N2pc does not necessarily imply that attention was not shifted to nonmatching cues, these studies nonetheless provide strong converging evidence against the notion that attention is allocated to cues that do not carry the target property and would thus appear to undermine the disengagement account.

Although the present results provide strong evidence against a two-process account of attentional capture, it is important to consider several potential limitations to this conclusion. First, the mixture analysis used in the present study involved comparing the predicted and observed variance for the intermediate distribution. There are, however, other means of assessing the presence of a mixture distribution. These include assessments of means, bimodality, and a fixed crossover point, as well as a more powerful technique called the multinomial maximum likelihood mixture (MMLM) analysis, which takes into account the overall shape of the distribution (see Yantis et al., 1991, for a discussion of the various mixture analyses). The MMLM analysis has an advantage over other mixture analysis methods, in that it provides the most comprehensive assessment. An appropriate circumstance for applying an MMLM analysis, however, is narrowly defined and difficult to obtain. Indeed, very few researchers have ever used an MMLM analysis with success. When the statistical power of an MMLM analysis was investigated using the present data, the data were found to be inappropriate for the analysis; the minimal statistical power suggested by Yantis et al. was not obtained. Research suggests that in the absence of the required statistical power for the MMLM, a mixture analysis using variability provides the best alternative. For example, Johnson and Yantis (1995) looked at predicted and observed variability in conjunction with an MMLM analysis, and the two tests converged on the same conclusion. Also, the advantage of the more comprehensive assessment provided by an MMLM analysis becomes particularly relevant when other, less broadly focused methods fail to reject the null hypothesis. In the present case, the null hypothesis was rejected, and therefore, the need for a more powerful test is obviated.

A second potential limitation on the conclusions of the mixture analysis concerns the validity of the assumption that the $100 \%$ and $0 \%$ target-color cue conditions reflect fully captured and not captured trials, respectively. This is especially true in the case of the $0 \%$ condition for several reasons. As was discussed above, there is some debate in the literature with respect to whether the lack of cue validity effects in this condition mean that attention was not captured or whether it reflects rapid disengagement and recovery such that the effects of capture are no longer evident at the 150 -msec SOA between cue and target (Theeuwes et al., 2000). As was argued above, the literature would appear to provide strong evidence against the rapid disengagement account. Moreover, even if the rapid disengagement account were true, it does not invalidate the mixture analysis (the primary purpose of the present study), because the $0 \%$ condition would represent trials on which attention has fully recovered from capture, which, for the purposes of a mixture analysis, is functionally equivalent to no-capture trials. Thus, even on a disengagement account, the present results show that variations in the magnitude of capture as a function of similarity do not reflect a mixture of fully captured and fully recovered trials, but, rather, reflect continuous variation in the speed of attentional disengagement.

One may also question the assumption of no capture in the $0 \%$ target-color cue condition on the basis of the presence of the significant negative cue validity effect for green targets in both experiments. Specifically, it might be argued that a significant cue validity effect, in either direction, implicates the allocation of spatial attention. Although this possibility cannot be ruled out with the existing data, it has been argued in previous studies that rather than reflecting the allocation of spatial attention, such effects may be a manifestation of top-down inhibitory processes that aid in the prevention of involuntary attentional allocation (Lamy et al., 2004). Nonetheless, given that the present mixture analysis rests critically on the assumption that the performance in the $0 \%$ target color reflects trials on which the capture of spatial attention does not occur, additional research is clearly needed to explore the precise nature of negative cuing effects.

Finally, note that although the present results disconfirm a two-process model in the context of color similarity effects in the spatial cuing paradigm in which targets and cues appear as singletons, it is unclear whether this pattern would generalize to situations in which cue and target displays are heterogeneous (see Bacon \& Egeth, 1994). Similarly, it is possible that other factors influencing the magnitude of attentional capture may operate under different rules. For example, as was noted above, Theeuwes (1990) found that variations in the magnitude of capture associated with relative salience (rather than similarity) may indeed conform to a two-process model. Future research is needed to address these interesting possibilities.

\section{AUTHOR NOTE}

B.A.A. is now at Johns Hopkins University. These experiments were conducted as part of the requirements for the Master of Science degree awarded to B.A.A. by Villanova University. The authors thank Steven Yantis for sharing his computer program for determining maximum likelihood mixing probabilities. Correspondence concerning this article should be addressed to C. L. Folk, Department of Psychology, Villanova, PA 19085 (e-mail: charles.folk@villanova.edu).

\section{REFERENCES}

Ansorge, U., \& Heumann, M. (2003). Top-down contingencies in peripheral cuing: The roles of color and location. Journal of Experimental Psychology: Human Perception \& Performance, 29, 937-948.

Bacon, W. F., \& EGETH, H. E. (1994). Overriding stimulus-driven attentional capture. Perception \& Psychophysics, 55, 485-496.

Burnham, B. R. (2007). Displaywide visual features associated with a search display's appearance can mediate attentional capture. Psychonomic Bulletin \& Review, 14, 392-422. 
Duncan, J., \& Humphreys, G. W. (1989). Visual search and stimulus similarity. Psychological Review, 96, 433-458.

EIMER, M., \& KISS, M. (2008). Involuntary attentional capture is determined by task set: Evidence from event-related brain potentials. Journal of Cognitive Neuroscience, 20, 1423-1433.

ERIKSEN, C. W., \& YEH, Y.-Y. (1985). Allocation of attention in the visual field. Journal of Experimental Psychology: Human Perception \& Performance, 11, 583-597.

Folk, C. L., Leber, A. B., \& Egeth, H. E. (2002). Made you blink! Contingent attentional capture produces a spatial blink. Perception \& Psychophysics, 64, 741-753.

Folk, C. L., \& Remington, R. W. (1998). Selectivity in distraction by irrelevant featural singletons: Evidence for two forms of attentional capture. Journal of Experimental Psychology: Human Perception \& Performance, 24, 847-858.

Folk, C. L., \& Remington, R. W. (2006). Top-down modulation of preattentive processing: Testing the recovery account of contingent capture. Visual Cognition, 14, 445-465.

FolK, C. L., \& Remington, R. W. (2008). Bottom-up priming of topdown attentional control settings. Visual Cognition, 16, 215-231.

Folk, C. L., Remington, R. W., \& Johnston, J. C. (1992). Involuntary covert orienting is contingent on attentional control settings. Journal of Experimental Psychology: Human Perception \& Performance, 18, 1030-1044.

Folk, C. L., Remington, R. W., \& Wright, J. H. (1994). The structure of attentional control: Contingent attentional capture by apparent motion, abrupt onset, and color. Journal of Experimental Psychology: Human Perception \& Performance, 20, 317-329.

Johnson, D. N., \& YANTIS, S. (1995). Allocating visual attention: Tests of a two-process model. Journal of Experimental Psychology: Human Perception \& Performance, 21, 1376-1390.

Jonides, J. (1980). Toward a model of the mind's eye's movement. Canadian Journal of Psychology, 34, 103-112.

JONIDES, J. (1981). Voluntary vs. automatic control over the mind's eye's movement. In J. B. Long \& A. D. Baddeley (Eds.), Attention and performance $I X$ (pp. 187-203). Hillsdale, NJ: Erlbaum.

JONIDES, J. (1983). Further toward a model of the mind's eye's movement. Bulletin of the Psychonomic Society, 21, 247-250.

Kiss, M., Jolicceur, P., Dell'Acqua, R., \& Eimer, M. (2008). Attentional capture by visual singletons is mediated by top-down task set: New evidence from the N2pc component. Psychophysiology, 45, 1013-1024.

Kiss, M., VAn Velzen, J., \& Eimer, M. (2008). The N2pc component and its links to attention shifts and spatially selective visual processing. Psychophysiology, 45, 240-249.
LAmy, D., Leber, A., \& Egeth, H. E. (2004). Effects of task relevance and stimulus-driven salience in feature-search mode. Journal of Experimental Psychology: Human Perception \& Performance, 30, 10191031.

Leblanc, E., Prime, D. J., \& Joliceur, P. (2008). Tracking the location of visuospatial attention in a contingent capture paradigm. Journal of Cognitive Neuroscience, 20, 657-671.

Lien, M.-C., Ruthruff, E., Goodin, Z., \& Remington, R. W. (2008). Contingent attentional capture by top-down control settings: Converging evidence from event-related potentials. Journal of Experimental Psychology: Human Perception \& Performance, 34, 509-530.

Posner, M. I. (1980). Orienting of attention. Quarterly Journal of Experimental Psychology, 32, 3-25.

Pratt, J., \& McAuliffe, J. (2002). Determining whether attentional control settings are inclusive or exclusive. Perception \& Psychophysics, 64, 1361-1370.

Theeuwes, J. (1990). Perceptual selectivity is task dependent: Evidence from selective search. Acta Psychologica, 74, 81-99.

Theeuwes, J. (1992). Perceptual selectivity for color and form. Perception \& Psychophysics, 51, 599-606.

TheEuwes, J. (1994). Stimulus-driven capture and attentional set: Selective search for color and visual abrupt onsets. Journal of Experimental Psychology: Human Perception \& Performance, 20, 799-806.

Theeuwes, J., Atchley, P., \& Kramer, A. F. (2000). On the time course of top-down and bottom-up control of visual attention. In S. Monsell \& J. Driver (Eds.), Control of cognitive processes: Attention and performance XVIII (pp. 105-124). Cambridge, MA: MIT Press.

Theeuwes, J., DeVries, G. J., \& Godijn, R. (2003). Attentional and oculomotor capture with static singletons. Perception \& Psychophysics, 65, 735-746.

Treisman, A. M., \& Gelade, G. (1980). A feature-integration theory of attention. Cognitive Psychology, 12, 97-136.

Wolfe, J. M., CAVE, K. R., \& Franzel, S. L. (1989). Guided search: An alternative to the feature integration model for visual search. Journal of Experimental Psychology: Human Perception \& Performance, 15 419-433.

Yantis, S., \& Jonides, J. (1984). Abrupt visual onsets and selective attention: Evidence from visual search. Journal of Experimental Psychology: Human Perception \& Performance, 10, 601-621.

Yantis, S., Meyer, D. E., \& Smith, J. E. K. (1991). Analyses of multinomial mixture distributions: New tests for stochastic models of cognition and action. Psychological Bulletin, 110, 350-374.

(Manuscript received June 10, 2009; revision accepted for publication September 11, 2009.) 